\title{
Igg Subclasses Targeting the Flagella of Salmonella Enterica Serovar Typhimurium Can Mediate Phagocytosis and Bacterial Killing
}

\author{
Yun Shan Goh ${ }^{1}$, Kathryn L Armour ${ }^{2}$, Michael R Clark ${ }^{2,3}$, Andrew J Grant ${ }^{1}$ and Pietro Mastroeni ${ }^{{ }^{*}}$ \\ ${ }^{1}$ Department of Veterinary Medicine, University of Cambridge, Madingley Road, Cambridge CB3 OES, United Kingdom \\ ${ }^{2}$ Department of Pathology, University of Cambridge, Tennis Court Road, Cambridge CB2 1QP, United Kingdom \\ ${ }^{3}$ Current address: Clark Antibodies Ltd, 10 Wellington Street, Cambridge CB1 1HW, United Kingdom
}

"Corresponding author: Dr. Pietro Mastroeni, Department of Veterinary Medicine, University of Cambridge, Madingley Road, Cambridge CB3 0ES, United Kingdom, Tel: +44-1223-765800; Fax: +44-1223-330848; E-mail: pm274@cam.ac.uk

Received date: May 16, 2016, Accepted date: May 26, 2016, Published date: May 30, 2016

Copyright: (c) 2016 Goh YS, et al. This is an open-access article distributed under the terms of the Creative Commons Attribution License, which permits unrestricted use, distribution, and reproduction in any medium, provided the original author and source are credited.

\begin{abstract}
Invasive non-typhoidal Salmonella are a common cause of invasive disease in immuno-compromised individuals and in children. Multi-drug resistance poses challenges to disease control, with a critical need for effective vaccines. Flagellin is an attractive vaccine candidate due to surface exposure and high epitope copy number, but its potential as a target for opsonophacytic antibodies is unclear.

We examined the effect of targeting flagella with different classes of IgG on the interaction between Salmonella Typhimurium and a human phagocyte-like cell line, THP-1. We tagged the FliC flagellar protein with a foreign CD52 mimotope (TSSPSAD) and bacteria were opsonized with a panel of humanised CD52 antibodies with the same antigen-binding $\mathrm{V}$-region, but different constant regions. We found that IgG binding to flagella increases bacterial phagocytosis and reduces viable intracellular bacterial numbers. Opsonisation with IgG3, followed by $\lg G 1$, IgG4, and $\lg$ 2, resulted in the highest level of bacterial uptake and in the highest reduction in the intracellular load of viable bacteria. Taken together, our data provide proof-of-principle evidence that targeting flagella with antibodies can increase the antibacterial function of host cells, with $\lg G 3$ being the most potent subclass. These data will assist the rational design of urgently needed, optimised vaccines against iNTS disease.
\end{abstract}

Keywords: Flagella; Antibody; Phagocytosis; IgG subclasses; Opsonisation

\section{Introduction}

Non-typhoidal Salmonella (NTS) disease is a major public health burden. It usually manifest as self-limiting gastroenteritis in humans [1]. However, in immuno-compromised individuals (such as malaria and HIV-infected patients) and children especially in developing countries, it predominantly manifests in an invasive NTS (iNTS) disease with bacteremia [2-4], which is most commonly caused by Salmonella enterica serovars Typhimurium and Enteritidis. The case fatality of iNTS is $20-25 \%$ in children [3] and up to $47 \%$ in HIVinfected adults [5]. Increased drug resistance and emergence of new multi-drug resistant $S$. enterica strains has made iNTS disease difficult to manage [6-8]. There is very broad consensus that vaccines against iNTS are urgently needed. Several classes of vaccines against iNTS (e.g. live attenuated, polysaccharide, conjugates) are currently being considered, but no vaccine is currently licensed for use in humans [9].

Antibodies and phagocytes are essential effectors that mediate protection against invasive salmonellosis [10-15]. Salmonella growth in the tissues is paralleled by the spread of the microorganisms throughout the body via the extracellular space and by blood from established infection foci to new sites [16]. Cytokine-driven host responses recruit phagocytes to multicellular pathological lesions, trapping the bacteria within discrete foci of infection [12-14,17], where the antimicrobial action of reactive oxygen and nitrogen species (ROS and RNS) restricts intracellular growth [17-20]. During their extracellular dispersion, Salmonella become vulnerable to antibodies and complement that opsonise the bacteria and target them to receptors on the surface of phagocytes, increasing the ROS-dependent antimicrobial functions of the host cells [12-15,17,21-23].

Infection with S. enterica induces production of antibodies against various bacterial targets such as flagellar proteins, outer membrane proteins, lipopolysaccharide (LPS), heat shock proteins and fimbriae [24-28]. However, the full spectrum of the antigen specificity of the protective antibody response against invasive salmonelloses is still unclear.

The surface exposed FliC flagellin protein is involved in bacterial invasion as illustrated by reduced invasiveness of FliC-deficient mutants in vitro [29], and contributes to bacterial virulence and protection in murine models of the disease [30-33]. Therefore, flagellin may be an attractive vaccine candidate. Flagellin could also serve as a carrier and an adjuvant. The flagellum is a polymer of flagellin and hence acts as a multivalent antigen expressing multiple copies of the epitope. Flagella have been used as a carrier for foreign antigens and have shown efficient antibody induction to both the flagellin and foreign epitopes [34-36]. Being surface exposed, both the flagellin and the foreign epitopes are efficiently presented to the immune system. The flagellin protein interacts with Toll-like receptor 5 (TLR-5), which activates NFKB [37]. This leads to the induction of a pro-inflammatory immune response, giving the flagella its adjuvanticity.

Given the increasing interest in the use of flagella as an antigen target and as a carrier [34-36, 38], we aimed to examine the potential efficacy of targeting the FliC protein with antibodies to enhance 
phagocyte functions known to be essential for protective immunity against Salmonella. We have previously shown that targeting the surface-abundant OmpA protein with human IgGs can increase the uptake and killing of bacteria by human phagocytic cells, the efficiency of these functions depending both on the subclass of the IgG and the FcR that is available for antibody binding [39]. Broadening the repertoire of antigens to be included in future iNTS multicomponent vaccines is desirable. Therefore, in the current study we used a similarly robust experimental system, where we tagged flagella with the CD52 mimotope (TSSPSAD) and then opsonised the bacteria with a panel of humanised CD52 antibodies that share the same antigenbinding V-region, but have constant regions of different subclasses. Using this system, we investigated whether targeting flagella with antibodies can mediate key phagocyte functions associated with host resistance to infection, in particular phagocytosis and intracellular bacterial killing.

\section{Materials and Methods}

\section{Reagents and media}

All reagents and media were obtained from Sigma-Aldrich, Poole, UK unless stated otherwise.

\section{Bacterial strains}

S. Typhimurium LDV321 [40] is a non-motile derivative of parent strain SL3261 [41], where fliC, fljA and $f l j B$ (but not the hin promoter) are excised. We generated a green fluorescent protein (GFP)expressing derivative of SL3261 by inserting a DNA fragment that consists of the gfp gene from Aequoria Victoria and a chloramphenicol resistance cassette between pseudogenes malX and malY on the chromosome by oligonucleotide-directed mutagenesis [42]. The DNA fragment consisting of the $g f p$ gene and the chloramphenicol resistance cassette with 5' and 3' arms homologous to the DNA flanking the pseudogenes was amplified by PCR using primer pair MalXT (5'-CCG CAG GTT CAG TCG GTA AAA GAT GAA ATG GTT GGC CTG ATG AAT ACC GTT CAG GCA TAA CCT GGG GTA ATG ACT CTC TAG C-3') and MalYCam (5'-CTA CGT ACA CCA TGT CCC GCG TCG GTC AAC TTC CTG TGA AAA ATC GAA CAT ATC CCT TCC GAC GTC ATT TCT GCC ATT CAT CC-3'). Underlined regions of the primers indicate sequences complementary to the downstream region of the malX gene and sequences complementary to the upstream region of the malY gene respectively. To allow tagging of the flagella, we transformed GFPexpressing $S$. Typhimurium LDV321 with a recombinant pFF408 $[40,43]$. The recombinant pFF408 was generated by inserting DNA encoding a short peptide (TSSPAD), which is a mimotope of the human CD52 antigen, between unique XhoI and BgIII restriction enzyme sites at the central region of the $f l i C$ gene in the plasmid pFF408 [43]. The $\mathrm{fliC}$ gene is under the native $\mathrm{fliC}$ promoter. To generate the fragment consisting of the sequence encoding for the TSSPSAD mimotope, we amplified a fragment upstream of the insertion site in pFF408 using primer pair CDPCRF3 (5'-CAT GAT TAC GAA TTC GTT ATC GGC-3') and CDPCRR3 (5'-TTT TTT CTC GAG ATC CGC GGA CGG GGA GGA GGT AGA TCT AGT ACC ACC AAG ACC AGT AGC-3'). Underlined segment of the CDPCRR3 primer encodes for the TSSPSAD mimotope, thereby inserting the mimotope to the fragment. This fragment that consisted of mimotope-encoding sequence was then inserted into pFF408 by conventional ligation. The insertion of the gfp gene between malX and
malY genes and the insertion of the TSSPSAD mimotope-coding sequence in the $\mathrm{fliC}$ gene were confirmed by standard sequencing. Expression of GFP and the mimotope were verified by immunofluorescence (see Supplementary Figure 1). We confirmed that the insertion of the CD52 mimotope did not interfere with assembly of the FliC flagella on the bacterial surface and the recombinant bacterial strain was motile (see Supplementary Figure 2).
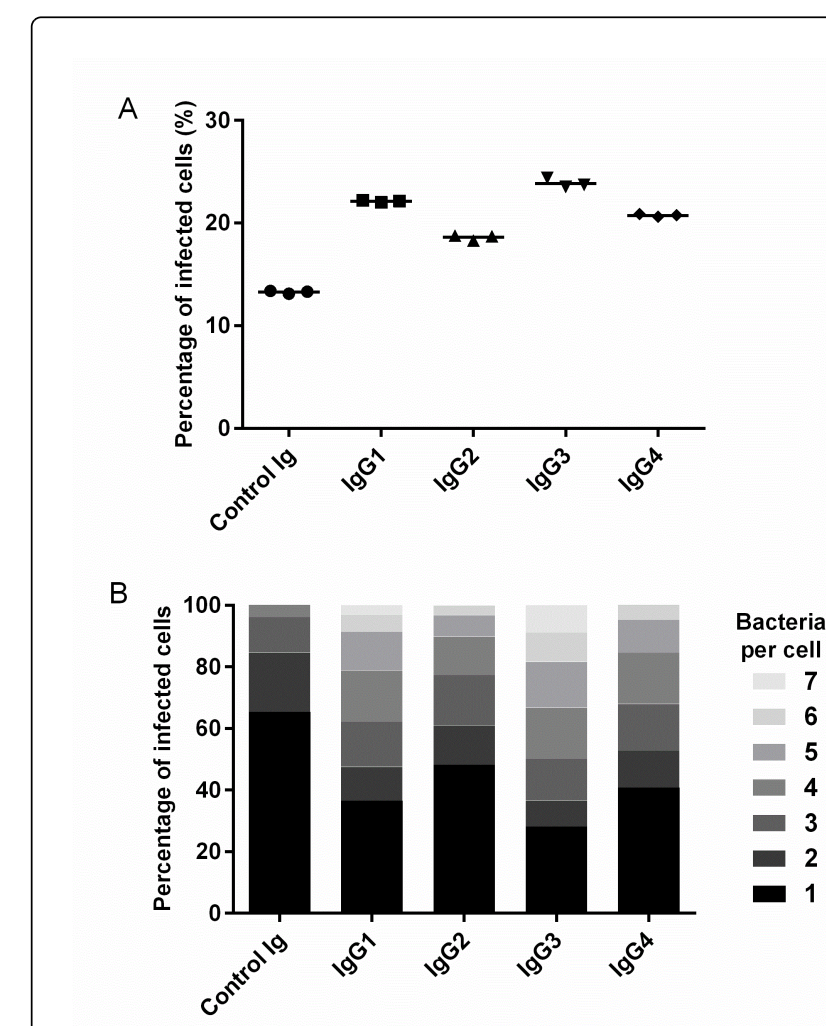

Figure 1: Bacterial uptake following infection of S. Typhimurium opsonised with antibodies targeting the bacterial flagella. THP-1 cells were infected with S. Typhimurium opsonised with antiTSSPSAD antibodies or control IgG. The anti-TSSPSAD antibodies were of different subclasses, either IgG1, IgG2, IgG3 or IgG4. (A) Percentage of infected cells. The graph shows the percentages of cells harbouring visible intracellular bacteria. Assay was performed as three independent biological replicates $(n=3)$, conducted in duplicate. Each data point in the graph represents the mean of duplicate for an individual experiment. The horizontal line in the graph represents the mean percentage of infected cells. 450 host cells were counted for each of the three independent set of experiments. (B) Intracellular bacterial distribution. The graph shows distributions of visible intracellular bacteria per infected cell, which are expressed as the mean distributions from three independent biological replicates. 450 host cells were counted for each of the three independent set of experiments.

\section{Cell culture}

The human monocyte cell line THP-1 was grown in RPMI-1640 supplemented with $10 \%$ fetal calf serum, $2 \mathrm{mM}$ L-glutamine, $0.05 \mathrm{mM}$ 2-mercaptoethanol at $37^{\circ} \mathrm{C}$. Prior to bacterial infection, THP-1 cells were grown in RPMI-1640 supplemented with $10 \% \mathrm{Nu}$ serum (VWR), 
$2 \mathrm{mM}$ L-glutamine, $0.05 \mathrm{mM}$ 2-mercaptoethanol for 22 days, followed by an incubation with $100 \mathrm{U} / \mathrm{ml} \mathrm{rIFN} \gamma$ for $48 \mathrm{~h}$ [44].

\section{Bacterial opsonisation}

Bacteria were opsonised as previously described [39]. Briefly, bacteria from an overnight culture were opsonised by incubation in either the humanised anti-TSSPSAD antibodies or the non-specific control antibody at a final concentration of $25 \mu \mathrm{g} / \mathrm{ml}$ (the highest concentration that does not agglutinate the bacteria) at $37^{\circ} \mathrm{C}$ with shaking for $30 \mathrm{~min}$. The humanised anti-TSSPSAD antibodies share the same variable regions (CAMPATH-1 [45]) that recognize the human CD52 mimotope, but are of different human antibody subclasses (either IgG1, IgG2, IgG3, or IgG4 $[46,47]$ ). The non-specific control antibody used in this study is the recombinant human Fog-1 IgG1 [47] antibody that recognizes the human RhD antigen. Unbound antibody was removed by extensive washing. Effective opsonisation was visualized by fluorescence microscopy using Alexa Fluor 568 conjugated goat anti-human IgG (Invitrogen).

\section{Immunofluorescence}

Following opsonisation at $37^{\circ} \mathrm{C}$ with shaking for 30 min with either the humanised anti-TSSPSAD antibodies or the non-specific control antibody at a final concentration of $25 \mu \mathrm{g} / \mathrm{ml}$, unbound antibody was removed by washing. The opsonized bacteria were then labeling with Alexa 568 conjugated goat anti-human $\operatorname{IgG}$ and visualized by fluorescence microscopy.

\section{Infection of THP-1 cells with $S$. Typhimurium}

Following opsonisation, the bacteria were added to the THP- 1 cells at multiplicity of infection (MOI) of 10:1 (bacteria: THP-1 cells) and incubated for $45 \mathrm{~min}$ at $37^{\circ} \mathrm{C}$ [39]. The infected cells were then washed five times with PBS. Any remaining extracellular bacteria were killed by incubating the infected cells with fresh culture medium containing $100 \mu \mathrm{g} / \mathrm{ml}$ gentamicin for $1 \mathrm{~h}$. This time point was taken as $0 \mathrm{~h}$. For time point $8 \mathrm{~h}$, the medium was replaced with fresh medium supplemented with $10 \mu \mathrm{g} / \mathrm{ml}$ gentamicin and the cells were incubated at $37^{\circ} \mathrm{C}$ for another $8 \mathrm{~h}$.

\section{Determination of the number of visible intracellular viable bacteria}

Twelve hours prior to infection, THP-1 cells were seeded onto polyL-Lysine treated coverslips (Fisher Scientific). THP-1 cells were infected with opsonised bacteria. At 0 h, THP-1 cells were fixed with $4 \%$ paraformaldehyde for $15 \mathrm{~min}$ before staining with mouse monoclonal anti-O4 antibodies (Abcam) and subsequently with secondary goat anti-mouse Alexa Fluor 405 antibody (Invitrogen). All antibodies were diluted 1:1000 in $10 \%$ normal goat serum (Dako). Coverslips were mounted onto Vecta bond-treated glass slides (Vector Laboratories) with mounting medium (Vectashield, Vector Laboratories) and viewed using a Leica DM6000B fluorescence microscope. Intracellular bacteria were discriminated from extracellular bacteria by the presence of GFP and the absence of labeling by the mouse monoclonal anti-O4 antibodies. For experiments exploring the percentage of infected cells and intracellular bacterial load distributions, 450 infected host cells were counted for each one of three repeats.

\section{Determination of intracellular bacterial viable counts}

At each time point, the infected THP-1 cells were harvested by lysing with $0.1 \%$ Triton-X for $15 \mathrm{~min}$. Viable bacterial colony-forming units (CFU) in the lysates were determined by the pour plate technique using LB agar.

\section{Host cell cytotoxicity}

Host cell death was determined using the Cytotox 96 nonradioactive cytotoxicity assay (Promega) according to manufacturer's protocol. The assay quantifies the amount of the cytosolic enzyme, lactate dehydrogenase ( $\mathrm{LDH})$, released into the supernatant when the membrane integrity of the cells was compromised as a result of cell death.

\section{Statistical analysis}

All sets of experiments were performed as three independent biological replicates $(n=3)$. For determination of intracellular viable bacterial counts and cell cytotoxicity, each independent set of experiment was carried out in technical duplicate. Comparisons between means were conducted using the paired Student's $t$-test. We considered every possible paired combination between groups and controlled for the familywise error rate using a Holm-Bonferroni correction. $\mathrm{P}$ value of $<0.05$ is considered to be significant. The data described in this study are all statistically significant.

\section{Results}

Targeting flagella with antibodies can enhance phagocytosis of $S$. Typhimurium by human cells

To examine whether antibodies that bind flagella can modulate bacterial uptake by human phagocytic cells, we used an approach that involves opsonisation of bacteria with recombinant humanised IgGs followed by the addition of the opsonised bacteria to the human phagocyte THP-1 cell line in vitro. We generated a recombinant $S$. Typhimurium LDV321-GFP strain where we inserted the CD52 mimotope TSSPSAD into FliC, allowing targeting by a panel of humanised anti-TSSPSAD antibodies with identical antigen-binding V-regions and different IgG constant regions (IgG1, IgG2, IgG3 and IgG4). Following a $45 \mathrm{~min}$ exposure to the opsonised bacteria, THP-1 cells were incubated for $1 \mathrm{~h}$ with culture medium containing $100 \mu \mathrm{g} / \mathrm{ml}$ of gentamicin to kill any extracellular bacteria. This is indicated as time point $0 \mathrm{~h}$. We used the percentage of infected cells and the numbers of intracellular bacteria per infected cell as robust parameters to determine the effect of anti-flagella antibodies on bacterial uptake.

Opsonisation with specific antibodies that target $S$. Typhimurium flagella resulted in a higher percentage of infected cells (Figure 1A), as compared to the non-specific control human IgG1 antibody. Comparing among the different antibodies, the percentages of infected cells were statistically different between all the IgG subclasses; the highest percentage was observed when the bacteria were opsonised with IgG3, followed by IgG1, IgG4 and IgG2. We then studied the effects of opsonisation on the intracellular bacterial loads. We determined the number of visible intracellular bacteria per infected cell by immunofluorescence. Extracellular bacteria were identified by immunolabelling with mouse monoclonal anti-O4 antibodies, while intracellular bacteria were identified by the presence of GFP and the absence of the mouse monoclonal anti-O: 4 antibody labeling. When the bacteria were opsonised with anti-TSSPSAD antibodies, we 
observed bacterial loads per phagocyte skewed towards higher intracellular densities (Figure 1B), as compared to the non-specific control antibody. Comparing among the different antibodies, the percentage of infected cells containing more than three bacteria was the highest when we used anti-TSSPSAD IgG3 antibodies to opsonize the bacteria (50\%), followed by IgG1 (38\%), IgG4 (32\%) and IgG2 (22\%). We considered every possible paired combination between the different antibody groups and controlled for the familywise error rate using a Holm-Bonferroni correction. The differences for all combination pairs of antibody (either with control isotype or with other isotype) were statistically significant.

Antibody binding to flagella can enhance the reduction in intracellular numbers of $S$. Typhimurium within human phagocytes

We have shown that antibodies targeting the flagella of $S$. Typhimurium via the TSSPSAD mimotope can enhance the uptake of $S$. Typhimurium by phagocytes. Next, we examined the effect of flagella-targeting antibodies on the numbers of viable intracellular bacteria. To explore this, we compared the intracellular viable bacterial counts from infected THP- 1 cell lysates at $0 \mathrm{~h}$ and at $8 \mathrm{~h}$ post infection (p.i.). This was determined by counting the numbers of viable intracellular bacteria in the infected THP-1 lysates at both time points by plating on agar.

Opsonisation with all subclasses of anti-TSSPSAD antibodies resulted in higher numbers of viable intracellular bacteria at $0 \mathrm{~h}$ p.i., as compared with the non-specific control antibody (Figure 2A). Comparing among the different antibodies, opsonisation with IgG3 resulted in the highest number of intracellular bacteria, followed by IgG1, IgG4 and IgG2 with statistically significant differences between all subclasses. At $8 \mathrm{~h}$ p.i., we observed lower intracellular viable bacterial counts for the bacteria opsonised with any of the antibodies, as compared with the counts at $0 \mathrm{~h}$ p.i. (Figure 2B). At $8 \mathrm{~h}$ p.i., the intracellular viable bacterial counts were the lowest in the cultures infected with bacteria opsonised with anti-TSSPSAD IgG3, followed by IgG1, IgG4, IgG2 and lastly, non-specific control antibody. We confirmed that the lower viable bacterial counts observed at $8 \mathrm{~h}$ p.i. were not due to a higher level of death of the infected THP-1 cells, which would result in exposure of the bacteria to the extracellular antibiotic (Figure S3). After $8 \mathrm{~h}$ of infection, opsonisation with IgG3 resulted in the lowest percentage of host cell death, followed by IgG1, then by IgG4 and non-specific control antibody, and lastly by IgG2. It is possible that, due to the higher efficiency in bacterial killing with IgG3 opsonisation, there is lower host cell death. At $0 \mathrm{~h}$ p.i., no significant levels of host cell death were observed.

We also calculated the magnitude of the reduction in intracellular bacterial numbers as the difference in the intracellular viable counts at $0 \mathrm{~h}$ and $8 \mathrm{~h}$ p.i. Opsonisation with specific antibodies targeting the flagella resulted in a greater reduction in the intracellular viable bacterial counts (Figure 2C), as compared to the non-specific control antibodies. When the bacteria were opsonised by anti-TSSPSAD antibodies, we observed a greater reduction in the intracellular viable bacterial counts with IgG3, followed by IgG1, IgG4 and IgG2.

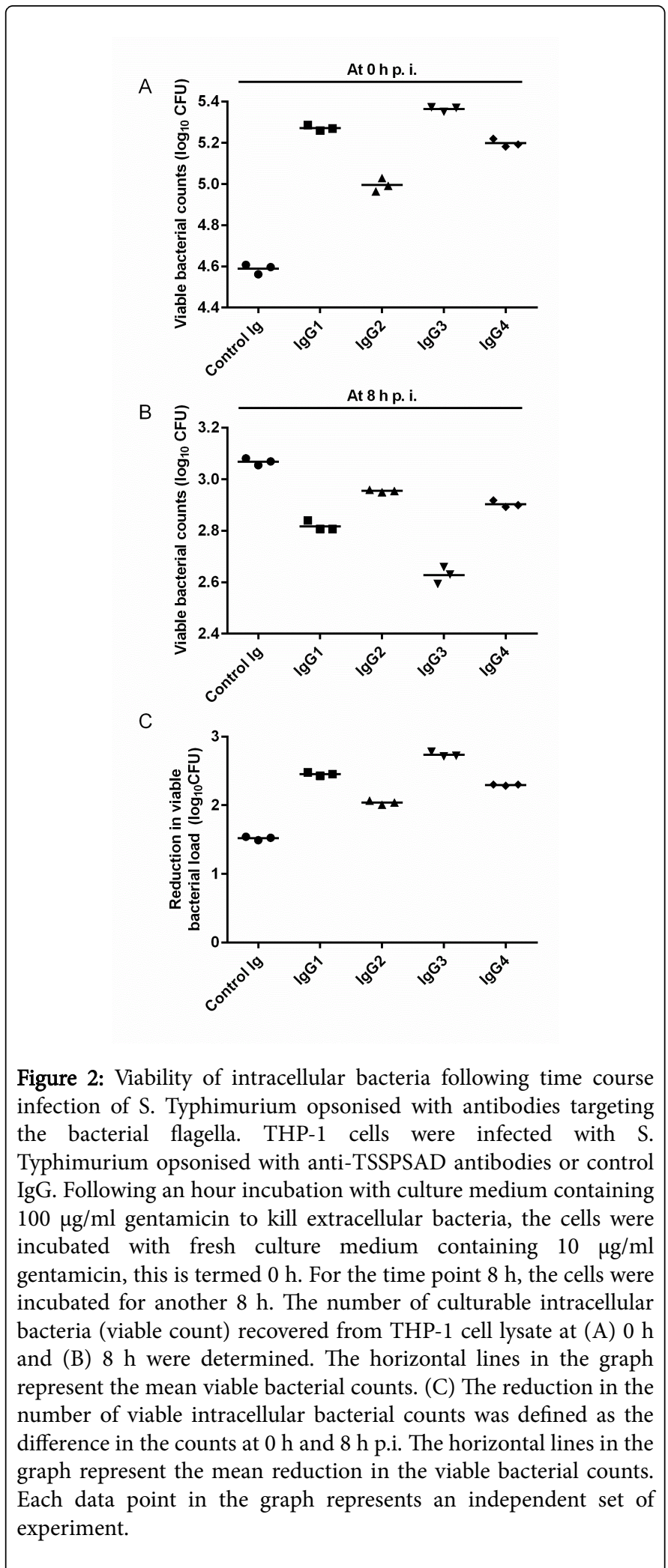

\section{Discussion}

Surface proteins to be good vaccine candidates need to be abundantly expressed, easily accessible to antibodies and can be easily 
purified in a conformation that retains immunogenicity. However these criteria do not guarantee that targeting a surface protein results in efficient enhancement of phagocyte functions, one of the main correlated of protection in invasive Salmonella infections. Therefore functional data on the effect of antibody binding to candidate surface antigens are essential for preclinical vaccine development. The Salmonella flagellum is a main bacterial target, inducing efficient antibody production upon infection [25-27]. Due to its surface localization, repetitive epitopes along their structure, and TLR-5associated pro-inflammatory adjuvanticity, flagella can be considered as good protein carriers for inclusion in conjugate vaccines against iNTS. However, to date, it is has not been clear whether antibodies against flagella can enhance opsonophagocytosis and killing of the bacteria by human phagocytes.

The key findings of this study are that: i) targeting flagella with antibodies enhances phagocytosis and subsequent reductions in intracellular loads of viable bacteria; and ii) different isotypes of human IgG differ in their ability to modulate phagocytosis and reductions in intracellular bacterial numbers. Opsonisation resulted in an increase in bacterial uptake, as observed with a higher percentage of infected cells, higher bacterial numbers per cell, and a higher number of viable intracellular bacterial counts at $0 \mathrm{~h}$ p.i. (as compared to the non-specific control isotype). Following the initial phagocytosis event, specific antibody opsonisation also resulted in a greater reduction in the number of viable intracellular bacterial counts at $8 \mathrm{~h}$ p.i. (as compared to the non-specific control isotype). The magnitude of this reduction in the observed $8 \mathrm{~h}$ time frame is indicative of bacterial killing by the host cells, although differences in the bacteriostatic functions of phagocytes may also play a minor role in the observed phenomena.

We found that the efficiency in mediating bacterial uptake and reductions in intracellular bacterial numbers by IgG targeting the flagella differs, depending on the IgG subclasses. IgG3 is more efficient than IgG1, followed by IgG4 and then IgG2. For all studied parameters of bacterial uptake (namely the percentage of infected cells, the intracellular bacterial distribution and the viable intracellular bacterial counts at $0 \mathrm{~h}$ p.i.), the increase of bacteria uptake was the greatest when S. Typhimurium was opsonised with IgG3, followed by IgG1, IgG4 and lastly, IgG2. We have observed the similar pattern between the $\operatorname{IgG}$ subclasses in intracellular bacterial killing at $8 \mathrm{~h}$ p.i.. One possible reason for the difference in the anti-bacterial killing ability between the different isotypes is the difference in the molecular flexibility of the different subclasses. The greater flexibility of the hinge region of IgG3 probably allows IgG3 to associate better with activating Fc $\gamma$ receptors such as Fc $\gamma$ RI and Fc $\gamma$ RIIA $[46,48,49]$ to mediate efficient bacterial uptake and the subsequent intracellular bacterial killing, possibly hence resulting in lower host cell death at $8 \mathrm{~h}$ p.i.. In fact, Th1associated IgG3 has been shown to be more advantageous than the other subclasses in other infection systems, where IgG3 is negatively associated with progression of clinical diseases [50]. We have recently demonstrated that Th1-associated murine IgG2a and IgG2b were the most efficient isotypes at lowering Salmonella bacterial load in the blood, liver and spleen in vivo following infection in mouse [51]. Taken together, this has implications in the formulation of the vaccine and the design of the vaccination regimes as the data suggest that formulations and regimes that induce a Thl response that is predominantly IgG3 antibody response could offer better protection against NTS.
In conclusion, our findings serve as a proof-of-principle, demonstrating that antibodies targeting flagella can enhance bacterial phagocytosis and intracellular bacterial killing against $S$. Typhimurium efficiently. Efficient uptake of the bacteria by phagocytic cells is particularly important as this could lead to efficient clearance of the bacteria from the bloodstream, and hence possibly reducing the occurrence of NTS bacteremia. In addition, the efficient intracellular bacterial killing by phagocytic cells is critical to containment of the infection which could lead to a faster resolution of the disease. Other studies have also illustrated a role for flagella in T-cell immunity and its association with protection in mice $[31,34,52,53]$.

Our study therefore support the notion that flagella-based vaccines, either as purified subunit protein preparations or as flagellapolysaccharide conjugates have the potential to be promising vaccine candidates for protection against NTS.

We have previously shown that antibodies targeting OmpA [39] and lipopolysaccharides (LPS) [51] can increase bacterial uptake and eventual bacterial killing. It is therefore interesting to speculate that combination vaccines eliciting the right IgG isotype profile against ompA, flagella and LPS antigens, possibly also though carefully chosen delivery systems, may have the potential of being highly protective against iNTS infections.

\section{Acknowledgement}

This work was supported by grants from the Wellcome Trust (081743/Z/06/Z) awarded to P.M. and A.J.G and from the Medical Research Council G0801161 awarded to A.J.G. and P.M. We would like to thank S.M.C. Newton for providing S. Typhimurium LDV321 and plasmid pFF408.

\section{References}

1. Boyle EC, Bishop JL, Grassl GA, Finlay BB (2007) Salmonella: from pathogenesis to therapeutics. J Bacteriol 189: 1489-1495.

2. MacLennan CA, M.M. Levine (2013) Invasive nontyphoidal Salmonella disease in Africa: current status. Expert Rev Anti Infect Ther 11: 443-446.

3. Feasey NA, Dougan G, Kingsley RA, Heyderman RS, Gordon MA (2012) Invasive non-typhoidal salmonella disease: an emerging and neglected tropical disease in Africa. Lancet 379: 2489-2499.

4. Graham SM (2010) Nontyphoidal salmonellosis in Africa. Curr Opin Infect Dis 23: 409-414.

5. Gordon MA, Banda HT, Gondwe M, Gordon SB, Boeree MJ, et al. (2002) Non-typhoidal salmonella bacteraemia among HIV-infected Malawian adults: high mortality and frequent recrudescence. Aids 16: 1633-1641.

6. Nielsen MV, Sarpong N, Krumkamp R, Dekker D, Loag W, et al. (2012) Incidence and characteristics of bacteremia among children in rural Ghana. PLoS One 7: e44063.

7. Tabu C, Breiman RF, Ochieng B, Aura B, Cosmas L, et al. (2012) Differing burden and epidemiology of non-Typhi Salmonella bacteremia in rural and urban Kenya, 2006-2009. PLoS One 7: e31237.

8. Crump JA, Sjölund-Karlssonb M, Gordonc MA, Parrye CM (2015) Epidemiology, Clinical Presentation, Laboratory Diagnosis, Antimicrobial Resistance, and Antimicrobial Management of Invasive Salmonella Infections. Clin Microbiol Rev 28: 901-937.

9. MacLennan CA, Martin LB, Micoli F (2014) Vaccines against invasive Salmonella disease: current status and future directions. Hum Vaccin Immunother 10: 1478-1493.

10. Gondwe EN, Molyneux ME, Goodall M, Graham SM, Mastroeni P, et al. (2010) Importance of antibody and complement for oxidative burst and killing of invasive nontyphoidal Salmonella by blood cells in Africans. Proc Natl Acad Sci U S A 107: 3070-3075. 
11. MacLennan CA (2014) Antibodies and protection against invasive salmonella disease. Front Immunol 5: 635.

12. Mastroeni P, Grant A (2013) Dynamics of spread of Salmonella enterica in the systemic compartment. Microbes Infect 15: 849-857.

13. Mastroeni P, Grant A, Restif O, Maskell D (2009) A dynamic view of the spread and intracellular distribution of Salmonella enterica. Nat Rev Microbiol 7: 73-80.

14. Mastroeni P, Grant AJ (2011) Spread of Salmonella enterica in the body during systemic infection: unravelling host and pathogen determinants. Expert Rev Mol Med 13: e12.

15. Mastroeni P, Villarreal-Ramos B, Hormaeche CE (1993) Adoptive transfer of immunity to oral challenge with virulent salmonellae in innately susceptible $\mathrm{BALB} / \mathrm{c}$ mice requires both immune serum and $\mathrm{T}$ cells. Infect Immun 61: 3981-3984.

16. Sheppard M, Webb C, Heath F, Mallows V, Emilianus R, et al. (2003) Dynamics of bacterial growth and distribution within the liver during Salmonella infection. Cell Microbiol 5: 593-600.

17. Grant AJ, Restif O, McKinley TJ, Sheppard M, Maskell DJ, et al. (2008) Modelling within-host spatiotemporal dynamics of invasive bacterial disease. PLoS Biol 6: e74.

18. Mastroeni P, Vazquez-Torres A, Fang FC, Xu Y, Khan S, et al. (2000) Antimicrobial actions of the NADPH phagocyte oxidase and inducible nitric oxide synthase in experimental salmonellosis. II. Effects on microbial proliferation and host survival in vivo. J Exp Med 192: 237-248.

19. Vazquez-Torres A, Jones-Carson J, Mastroeni P, Ischiropoulos H, Fanga FC (2000) Antimicrobial actions of the NADPH phagocyte oxidase and inducible nitric oxide synthase in experimental salmonellosis. I. Effects on microbial killing by activated peritoneal macrophages in vitro. J Exp Med 192: 227-236.

20. Vazquez-Torres A, Xu Y, Jones-Carson J, Holden DW, Lucia SM, et al. (2000) Salmonella pathogenicity island 2-dependent evasion of the phagocyte NADPH oxidase. Science 287: 1655-1658.

21. Liang-Takasaki CJ, Saxén H, Mäkelä PH, Leive L (1983) Complement activation by polysaccharide of lipopolysaccharide: an important virulence determinant of salmonellae. Infect Immun 41: 563-569.

22. Menager N, Foster G, Ugrinovic S, Uppington H, Verbeek S, et al. (2007) $\mathrm{Fc} \gamma$ receptors are crucial for the expression of acquired resistance to virulent Salmonella enterica serovar Typhimurium in vivo but are not required for the induction of humoral or T-cell-mediated immunity. Immunology 120: 424-432.

23. Uppington H, Menager N, Boross P, Wood J, Sheppard M, et al. (2006) Effect of immune serum and role of individual Fc $\gamma$ receptors on the intracellular distribution and survival of Salmonella enterica serovar Typhimurium in murine macrophages. Immunology 119: 147-158.

24. Calderón I, Lobos SR, Rojas HA, Palomino C, Rodríguez LH, et al. (1986) Antibodies to porin antigens of Salmonella typhi induced during typhoid infection in humans. Infect Immun 52: 209-212.

25. Brown A, Hormaeche CE (1989) The antibody response to salmonellae in mice and humans studied by immunoblots and ELISA. Microb Pathog 6: 445-454.

26. Sztein MB, Wasserman SS, Tacket CO, Edelman R, Hone D, et al. (1994) Cytokine production patterns and lymphoproliferative responses in volunteers orally immunized with attenuated vaccine strains of Salmonella typhi. J Infect Dis170: 1508-1517.

27. Cooper GL, Thorns CJ (1996) Evaluation of SEF14 fimbrial dot blot and flagellar western blot tests as indicators of Salmonella enteritidis infection in chickens. Vet Rec 138: 149-153.

28. Harrison JA, Villarreal-Ramos B, Mastroeni P, Demarco de Hormaeche R, Hormaeche CE (1997) Correlates of protection induced by live AroSalmonella typhimurium vaccines in the murine typhoid model. Immunology 90: 618-625.

29. La Ragione RM, Cooley WA, Velge P, Jepson MA, Woodward MJ (2003) Membrane ruffling and invasion of human and avian cell lines is reduced for aflagellate mutants of Salmonella enterica serotype Enteritidis. Int J Med Microbiol 293: 261-272.
30. Ikeda JS, Schmitt CK, Darnell SC, Watson PR, Bispham J, et al. (2001) Flagellar phase variation of Salmonella enterica serovar Typhimurium contributes to virulence in the murine typhoid infection model but does not influence Salmonella-induced enteropathogenesis. Infect Immun 69: 3021-3030.

31. Gat O, Galen JE, Tennant S, Simon R, Blackwelder WC, et al. (2011) Cellassociated flagella enhance the protection conferred by mucosallyadministered attenuated Salmonella Paratyphi A vaccines. PLoS Negl Trop Dis 5: el373.

32. Simon R, Tennant SM, Wang JY, Schmidlein PJ, Lees A, et al. (2011) Salmonella enterica serovar enteritidis core $O$ polysaccharide conjugated to $\mathrm{H}: \mathrm{g}, \mathrm{m}$ flagellin as a candidate vaccine for protection against invasive infection with S. enteritidis. Infect Immun 79: 4240-4249.

33. Simon R, Wang JY, Boyd MA, Tulapurkar ME, Ramachandran G, et al. (2013) Sustained protection in mice immunized with fractional doses of Salmonella Enteritidis core and $\mathrm{O}$ polysaccharide-flagellin glycoconjugates. PLoS One 8: e64680.

34. Braga CJ, Massis LM, Sbrogio-Almeida ME, Alencar BC, Bargieri DY, et al. (2010) CD8+ T cell adjuvant effects of Salmonella FliCd flagellin in live vaccine vectors or as purified protein. Vaccine 28: 1373-1382.

35. Stocker BA, Newton SM (1994) Immune responses to epitopes inserted in Salmonella flagellin. Int Rev Immunol 11: 167-178.

36. Verma NK, Ziegler HK, Stocker BA, Schoolnik GK (1995) Induction of a cellular immune response to a defined T-cell epitope as an insert in the flagellin of a live vaccine strain of Salmonella. Vaccine 13: 235-244.

37. Simon R, Samuel CE (2007) Innate interferon response in macrophage and epithelial cells infected with wild-type compared to DNA adenine methylase and flagellin mutant Salmonella enterica serovar Typhimurium. J Interferon Cytokine Res 27: 317-327.

38. Eom JS, Seok Kim J, Im Jang J, Kim BH, Young Yoo S, et al. (2013) Enhancement of host immune responses by oral vaccination to Salmonella enterica serovar Typhimurium harboring both FliC and FljB flagella. PLoS One 8: e74850.

39. Goh YS, Grant AJ, Restif O, McKinley TJ, Armour KL, et al. (2011) Human IgG isotypes and activating Fcgamma receptors in the interaction of Salmonella enterica serovar Typhimurium with phagocytic cells. Immunology 133: 74-83.

40. Massis LM, Braga CJ, Sbrogio-Almeida ME, Lauand C, Newton SM, et al. (2008) Anti-flagellin antibody responses elicited in mice orally immunized with attenuated Salmonella enterica serovar Typhimurium vaccine strains. Mem Inst Oswaldo Cruz 103: 606-610.

41. Hoiseth SK, Stocker BA (1981) Aromatic-dependent Salmonella typhimurium are non-virulent and effective as live vaccines. Nature 291: 238-239.

42. Hautefort I, Proença MJ, Hinton JC (2003) Single-copy green fluorescent protein gene fusions allow accurate measurement of Salmonella gene expression in vitro and during infection of mammalian cells. Appl Environ Microbiol 69: 7480-7491.

43. Newton SM, Jacob CO, Stocker BA (1989) Immune response to cholera toxin epitope inserted in Salmonella flagellin. Science 244: 70-72.

44. Shen L, Graziano RF, Fanger MW (1989) The functional properties of Fc gamma RI, II and III on myeloid cells: a comparative study of killing of erythrocytes and tumor cells mediated through the different $\mathrm{Fc}$ receptors. Mol Immunol 26: 959-969.

45. Riechmann L, Clark M, Waldmann H, Winter G (1988) Reshaping human antibodies for therapy. Nature 332: 323-327.

46. Redpath S, Michaelsen TE, Sandlie I, Clark MR (1998) The influence of the hinge region length in binding of human IgG to human Fcgamma receptors. Hum Immunol 59: 720-727.

47. Armour KL, Clark MR, Hadley AG, Williamson LM (1999) Recombinant human IgG molecules lacking Fcgamma receptor I binding and monocyte triggering activities. Eur J Immunol 29: 2613-2624.

48. Roux KH, Strelets L, Michaelsen TE (1997) Flexibility of human IgG subclasses. J Immunol 159: 3372-3382. 
Citation: Goh YS, Armour KL, Clark MR, Grant AJ, Mastroeni P (2016) Igg Subclasses Targeting the Flagella of Salmonella Enterica Serovar Typhimurium Can Mediate Phagocytosis and Bacterial Killing . J Vaccines Vaccin 7: 322. doi:10.4172/2157-7560.1000322

Page 7 of 7

49. Burton DR, Woof JM (1992) Human antibody effector function. Adv Immunol 51: 1-84.

50. Taylor RR, Allen SJ, Greenwood BM, Riley EM (1998) IgG3 antibodies to Plasmodium falciparum merozoite surface protein 2 (MSP2): increasing prevalence with age and association with clinical immunity to malaria. Am J Trop Med Hyg 58: 406-413.

51. Goh YS, Clare S, Micoli F, Saul A, Mastroeni P, et al. (2015) Monoclonal Antibodies of a Diverse Isotype Induced by an O-Antigen Glycoconjugate Vaccine Mediate In Vitro and In Vivo Killing of African Invasive Nontyphoidal Salmonella. Infect Immun 83: 3722-3731.
52. Johanns TM, Ertelt JM, Lai JC, Rowe JH, Avant RA, et al. (2010) Naturally occurring altered peptide ligands control Salmonella-specific CD4+ $\mathrm{T}$ cell proliferation, IFN-gamma production, and protective potency. J Immunol 8: 869-876.

53. McSorley SJ, Cookson BT, Jenkins MK (2000) Characterization of CD4+ T Cell Responses During Natural Infection with Salmonella typhimurium. J Immunol 164: 986-993. 